\title{
Epsin 3 potentiates the NF-кB signaling pathway to regulate apoptosis in breast cancer
}

\author{
QIANXUE WU, QING LI, WENMING ZHU, XIANG ZHANG and HONGYUAN LI \\ Department of Endocrine and Breast Surgery, The First Affiliated Hospital of Chongqing Medical University, \\ Chongqing Medical University, Chongqing 400016, P.R. China
}

Received January 26, 2021; Accepted September 22, 2021

DOI: $10.3892 / \mathrm{mmr} .2021 .12531$

\begin{abstract}
Endocrine drug resistance is common in some patients with estrogen receptor (ER)-positive breast cancer, so it is necessary to identify potential therapeutic targets. The aim of the present study was to investigate the regulatory effect and mechanism of epsin 3 (EPN3) expression level changes on the proliferation and apoptosis of ER-positive breast cancer. Online GEPIA was used to analyze the expression level of EPN3 in breast cancer. The online Kaplan-Meier plotter tool was used to analyze the relationship between EPN3 expression and the prognosis of patients with breast cancer. Reverse transcription-quantitative PCR, immunohistochemistry and western blotting were performed to detect the mRNA and protein expression levels of EPN3 in breast cancer tissues and cells. A lentiviral infection system was used to knockdown the expression of EPN3 in breast cancer cell lines. Cell Counting Kit- 8 and flow cytometry assays were conducted to detect the effect of EPN3 knockdown on breast cancer cell proliferation and apoptosis. Western blotting was used to detect the regulation of EPN3 expression on NF- $\mathrm{KB}$, and immunofluorescence was performed to detect the effect of EPN3 expression on NF- $\mathrm{kB}$ nuclear translocation. The results demonstrated that the expression level of EPN3 in breast cancer tissues was higher compared with that in adjacent tissues $(\mathrm{P}<0.05)$. The expression level of EPN3 in the ER-positive breast cancer cell line, MCF7, was higher compared with that in the other cell lines (MCF10A, ZR75-1, MDA-MB-231, BT549 and SK-BR-3). After knocking down the expression of EPN3 in MCF7 cells, the proliferative ability of the cells was decreased, and the apoptosis rate was increased $(\mathrm{P}<0.05)$. After EPN3 knockdown
\end{abstract}

Correspondence to: Professor Hongyuan Li or Dr Xiang Zhang, Department of Endocrine and Breast Surgery, The First Affiliated Hospital of Chongqing Medical University, Chongqing Medical University, 1 Youyi Road, Chongqing 400016, P.R. China

E-mail: hongy_li@hotmail.com

E-mail: cq_zhangxiang@163.com

Key words: epsin 3, estrogen receptor-positive breast cancer, $\mathrm{NF}-\kappa \mathrm{B}$, apoptosis in MCF7 cells, the phosphorylation of NF- $\mathrm{NB}$ was decreased $(\mathrm{P}<0.05)$, and the nuclear translocation signal was weakened. Thus, it was suggested that EPN3 promoted cell proliferation and inhibited cell apoptosis by regulating the NF- $\mathrm{KB}$ signaling pathway in ER-positive breast cancer.

\section{Introduction}

Breast cancer is one of the most common malignant tumors in women and seriously threatens women's health (1). In 2019, 268,600 new cases of invasive breast cancer and 48,100 cases of ductal carcinoma in situ were diagnosed among US women, and 41,760 women died from this disease. Estrogen receptor (ER)-positive breast cancer accounts for $\sim 2 / 3$ of all breast cancers (2). Compared with other subtypes of breast cancer, patients with ER-positive breast cancer have a good prognosis (3). However, due to the phenomenon of endocrine resistance, patients with ER-positive breast cancer often succumb to the disease (4). In addition, although patients with ER-positive breast cancer receive adjuvant postoperative treatment, the rate of tumor recurrence within 5-10 years after surgery remains high (5). Research at the genetic level can not only redefine cancer, but also bring significant prospects for cancer treatment, such as targeting mutant p53, for efficient cancer therapy (6). Therefore, the discovery of molecular biological mechanism-related genes in ER-positive breast cancer is helpful for the development of treatment strategies.

The epsin family includes epsin 1 (EPN1), EPN2 and EPN3, which belong to the family of endocytic clathrins and are involved in receptor endocytosis (7). Although EPN1 and EPN2 are widely and highly expressed in tumors, and are involved in regulating biological functions such as angiogenesis (8), there are few studies on EPN3 in cancer. Wang et al (9) reported that EPN3 was involved in the regulation of glioma invasion and metastasis. It has also been revealed that EPN3 can be used as a downstream target of p53 to participate in the regulation of tumor cell senescence and apoptosis (10). However, to the best of our knowledge, EPN3 has rarely been studied in breast cancer. Previous studies have shown that EPN3 was an oncogene associated with the prognosis and treatment of breast cancer, and that it can promote breast cancer metastasis via epithelial-mesenchymal transition regulation (11). However, the function and mechanism of EPN3 in regulating 
cell proliferation and apoptosis in ER-positive breast cancer have not been completely elucidated.

The present study aimed to investigate the biological function and mechanism of EPN3 in regulating cell proliferation and apoptosis in ER-positive breast cancer, and to provide new insights into the role of EPN3 in promoting the occurrence and development of ER-positive breast cancer.

\section{Materials and methods}

Cell lines and tissue specimens. The human breast cancer cell lines, MCF7, ZR75-1, MDA-MB-231, BT549 and SK-BR-3, and the normal mammary epithelial cell line, MCF10A, were obtained from the American Type Culture Collection.

Human breast cancer samples $(n=33)$ and adjacent normal tissues $(n=33)$ for immunohistochemical staining and reverse transcription-quantitative PCR (RT-qPCR) were obtained from The First Affiliated Hospital of Chongqing Medical University between March 2019 and August 2019. The use of human specimens was approved by the Institutional Ethics Committee of The First Affiliated Hospital of Chongqing Medical University (approval no. IRB2021-181-01). The written informed consent of the patients has been obtained. All patients were female, aged 28-65 years. The distance between cancer tissue and adjacent normal tissue was $5 \mathrm{~cm}$. All patients were newly diagnosed with breast cancer. Patients who had received neoadjuvant chemotherapy or neoadjuvant endocrine therapy were excluded.

Antibodies and reagents. FBS, RPMI-1640 medium, penicillin/streptomycin and $0.25 \%$ trypsin were purchased from Biological Industries. The rabbit EPN3 polyclonal antibody (cat. no. ab185577) was purchased from Abcam. Anti-NF-кB (cat. no. 8242), anti-phosphorylated (p)-NF-кB (cat. no. 3033), anti-caspase 9 (cat. no. 9504), anti-cleaved-caspase 9 (cat. no. 9509), anti-caspase 3 (cat. no. 9662), anti-cleaved-caspase 3 (cat. no. 9664), anti-cleaved-caspase 7 (cat. no. 9491), anti-Bcl-2 (cat. no. 4223), anti-Bid (cat. no. 2002), anti-Bad (cat. no. 9239), anti-mouse IgG, HRP-linked antibody (cat. no. 7076), and anti-rabbit IgG, HRP-linked Antibody (cat. no. 7074) were all purchased from Cell Signaling Technology, Inc. GAPDH antibody (cat. no. GB11002) was purchased from Wuhan Servicebio Technology Co., Ltd. RNAisoPlus (cat. no. 9109), cDNA reverse transcription kit (cat. no. RR047A) and SYBR (cat. no. RR820A) were purchased from Takara Bio, Inc. Nude mice were purchased from Charles River Laboratories (Beijing, China). Estradiol cypionate (cat. no. HY-B1100) was purchased from MedChemExpress. Lipofectamine ${ }^{\circledR} 2000$ was purchased from Invitrogen (Thermo Fisher Scientific, Inc.). The Cell Counting Kit (CCK)-8 assay (cat. no. CK04) was purchased from Dojindo Laboratories, Inc. ECL hypersensitive photoluminescence solution (cat. no. WLA006b) was purchased from Wanleibio Co., Ltd. Immunostaining permeable solution (cat. no. P0096), immunostaining blocking solution (cat. no. P0102), FITC-labeled goat anti-rabbit IgG (Beyotime) (cat. no. A0562), DAPI (cat. no. C1002), RIPA lysis buffer (cat. no. P0013B), anti-fluorescence quenching tablet (cat. no. P0126) and BCA kit (cat. no. P0012S) were purchased from Beyotime Institute of Biotechnology.
Cell culture. The human breast cancer cell lines were cultured in RPMI-1640 medium containing 10\% FBS and $1 \%$ penicillin/streptomycin, in a $37^{\circ} \mathrm{C}$ cell incubator containing $5 \% \mathrm{CO}_{2}$. The medium was changed once every 2-3 days. MCF10A cells were cultured in DMEM/F12 supplemented with insulin $(10 \mu \mathrm{g} / \mathrm{ml})$, epidermal growth factor (20 ng/ml), $100 \mathrm{ng} / \mathrm{ml}$ cholera virus, $0.5 \mu \mathrm{g} / \mathrm{ml}$ hydrocortisone and 5\% horse serum (Gibco; Thermo Fisher Scientific, Inc.) as described previously (11).

Lentiviral transfection. The two short hairpin RNAs (shRNA/sh) were designed on the biosettia website (biosettia. com/support/shrna-designer), synthesized by Shanghai GenePharma Co., Ltd. and cloned into the pLVX-shRNA1-puro (You Bio) vector to generate the pLV-shRNA vector. The lentivirus shRNA sequence was as follows: sh-EPN3\#1, 5'-GAC CTTTGACCCATTTGCCAAT-3'; and sh-EPN3\#2, 5'-GCT GTCACTCAATTCGGTCATT-3'. According to the manufacturer's instructions, Lipofectamine 2000 was used to transfect the lentiviral vector $(20 \mu \mathrm{g}, \operatorname{PxpAx} 2: \mathrm{PMD} 2 \mathrm{~g}$ :pLVX=3:1:4) into $293 \mathrm{~T}$ cells (Thermo Fisher Scientific) at $37^{\circ} \mathrm{C}$ for $48 \mathrm{~h}$, and generate the 2 nd generation lentivirus packaging system by transfecting the packaging plasmid with calcium phosphate. $48 \mathrm{~h}$ after transfection, the supernatant was collected in a $15 \mathrm{ml}$ centrifuge tube and centrifuged at 3,000 rpm for $20 \mathrm{~min}$. The supernatant was filtered with a $0.45 \mu \mathrm{m}$ filter and stored at $-80^{\circ} \mathrm{C}$. When the breast cancer cell density reached $30-50 \%$, the cells were infected with lentivirus according to the manufacturer's instructions at $37^{\circ} \mathrm{C}$. At $48 \mathrm{~h}$ after transfection, the stable cell lines shEPN3\#1 and shEPN3\#2 were selected by using puromycin for 48-72 h. The follow-up experiment was carried out after continuous use of puromycin for 10 days. The concentration of puromycin used for selection was $2 \mu \mathrm{g} / \mathrm{ml}$, and the concentration used for maintenance was $1 \mu \mathrm{g} / \mathrm{ml}$. The cells infected with the blank control virus were designated as the control group. Extraction of total protein and detection of knockdown efficiency was then performed.

Immunohistochemistry (IHC). Human breast cancer samples, adjacent normal and xenograft tumor tissue were used for IHC staining. The samples were fixed with $4 \%$ paraformaldehyde at room temperature for $24 \mathrm{~h}$. IHC was performed according to the manufacturer's instructions (cat. no. SP9001; ZSGB-BIO). The thickness of tissue section was $4 \mu \mathrm{m}$. The tissue sections were dewaxed and the antigen was repaired in $95^{\circ} \mathrm{C}$ citrate buffer (pH6.0) for $20 \mathrm{~min}$ and cooled to room temperature. The slides were immersed in $3 \%$ peroxidase solution at room temperature for $20 \mathrm{~min}$. After blocking with $10 \%$ normal goat serum (cat. no. SP9001; ZSGB-BIO) at room temperature for 30 min and washing with TBS-10\% Tween-20 three times, the sections were incubated with the primary antibody (anti-EPN3, dilution, 1:200) against the target protein overnight at $4^{\circ} \mathrm{C}$. After washing with TBS-10\% Tween-20 three times, the slices were incubated with biotinylated goat anti-mouse IgG (dilution, 1:25) at room temperature for $30 \mathrm{~min}$, washed with TBS-10\% Tween-20 three times and incubated with HRP-coupled with streptavidin-biotin at room temperature for $30 \mathrm{~min}$. After washing with TBS-10\% Tween-20 three times, the slices were stained with DAB at room temperature for 1 min under an optical microscope (Trinocular Biological 
Microscope, CX43, Olympus, x10 magnification), re-stained with hematoxylin at room temperature for $10 \mathrm{sec}$, dehydrated and sealed with neutral resin (cat. no. G8590, Solarbio) at room temperature for $20 \mathrm{~min}$. The protein expression level was determined according to the staining intensity and the percentage of immunoreactive cells.

Cell proliferation assay. In total, 2,000 cells from each group (sh-NC or sh-MCF7 goup) were inoculated in 96-well culture plates. At the indicated time points ( $0,24,48$ and $72 \mathrm{~h}), \mathrm{CCK}-8$ reagent was used according to the manufacturer's instructions. The duration of incubation with $\mathrm{CCK}-8$ reagent at $37^{\circ} \mathrm{C}$ was $2 \mathrm{~h}$. The absorbance values were measured using a microplate reader (Infinite 200 PRO; Tecan Group, Ltd.) at $450 \mathrm{~nm}$.

Cell cycle analysis. Cells were washed twice with PBS and fixed in $70 \%$ cold ethanol at $4{ }^{\circ} \mathrm{C}$ for $30 \mathrm{~min}$. The cells were rewashed with PBS and incubated with $100 \mu \mathrm{l}$ RNase A $(0.1 \mathrm{mg} / \mathrm{ml})$ and $2 \mu \mathrm{l}$ PI (Sigma-Aldrich; Merck KGaA) for $30 \mathrm{~min}$ at $37^{\circ} \mathrm{C}$ in the dark. Cell cycle distribution was analyzed via flow cytometry (BD CellQuest Pro, version 5.1) using FACS Calibur (BD Biosciences).

Apoptosis analysis. In total, $\sim 1 \times 10^{6}$ cells in each group were washed with PBS and suspended in $100 \mu \mathrm{l}$ binding buffer. After incubation with Annexin V-FITC $(20 \mu \mathrm{g} / \mathrm{ml})$ and PI $(50 \mu \mathrm{g} / \mathrm{ml})$ (both Sigma-Aldrich; Merck KGaA) at room temperature for $30 \mathrm{~min}$, apoptosis was detected using a FACSCalibur (BD Biosciences) flow cytometer (BD CellQuest Pro, version 5.1). Total cell apoptosis was calculated as the percentage of early + late apoptotic cells.

Western blotting. All cells were lysed in RIPA buffer to obtain total proteins. The protein was extracted and the concentration was measured using a BCA protein kit. The same amount of denatured protein $(40 \mu \mathrm{g})$ and $10 \%$ SDS-PAGE were used for electrophoresis and then electrotransferred to the PVDF membrane and sealed in 5\% skimmed milk for $30 \mathrm{~min}$ at room temperature. The membrane was incubated with the primary antibody [anti-NF- $\kappa$ B, anti-phosphorylated (p)-NF- $\kappa$ B, anti-caspase 9, anti-cleaved-caspase 9, anti-caspase 3, anti-cleaved-caspase 3, anti-cleaved-caspase 7, anti-Bcl-2, anti-Bid, anti-Bad, anti-EPN3, and anti-GAPDH] overnight at $4^{\circ} \mathrm{C}$, and then with the second antibody (anti-rabbit IgG or anti-mouse $\mathrm{IgG}$ ) at room temperature for $1 \mathrm{~h}$. The Fusion FX7 Spectra multifunction imaging system was used to detect bands. The experiment was repeated three times. The dilution of the primary antibody was 1:1,000 and that of the second antibody was 1:2,000 (12).

$R T$ - $q P C R$. Total RNA was extracted using RNAiso Plus reagent (Takara Bio, Inc.) according to the manufacturer's instructions. RNA was then reverse transcribed to cDNA using a RT kit (Takara Bio, Inc.). The kit was used according to the manufacturer's instructions. TB GREEN (Takara Bio, Inc.) was used for further operations. The expression levels of the designated genes and GAPDH were determined using an ABI 7500 Real-Time PCR system (Applied Biosystems; Thermo Fisher Scientific, Inc.). The thermocycling conditions were as follows: Initial denaturation: $95^{\circ} \mathrm{C}$ for $30 \mathrm{sec}$, followed by 40 of cycles of denaturation, annealing, elongation and final extension $\left(95^{\circ} \mathrm{C}\right.$ for $5 \mathrm{sec}$ and $60^{\circ} \mathrm{C}$ for $\left.30 \mathrm{sec}\right)$. The primer sequences were as follows: GAPDH sense, 5'-CAATGACCC CTTCATTGACC-3' and antisense, 5'-GACAAGCTTCCC GTTCTCAG-3'; and EPN3 sense, 5'-GACATCTTCGTACCT GCCCTG-3' and antisense, 5'-TCTGTGTTCGGCCTAAAA CCT-3'. All reactions were performed in triplicate. GAPDH was used as an internal reference, and the relative expression of target gene was calculated using the $2^{-\Delta \Delta \mathrm{Cq}}$ method (13).

Immunofluorescence. In total, $\sim 1 \times 10^{4}$ cells seeded into plates were attached to slides in 24-well plates, and the following day, the cell climbing slices were washed twice with PBS and fixed in $4 \%$ paraformaldehyde for $5 \mathrm{~min}$ at room temperature. After permeation and sealing, the cell climbing slices were incubated

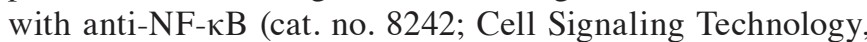
Inc.; 1:200) overnight at $4^{\circ} \mathrm{C}$. After washing with PBS, they were incubated with goat anti-rabbit IgG labeled with FITC for $3 \mathrm{~h}$ at room temperature. DAPI staining was performed for $5 \mathrm{~min}$ at room temperature, and $3 \mu \mathrm{l}$ anti-fluorescence quenching solution was used to seal the slices on the glass slides. An immunofluorescence microscope (Zeiss camera; Zeiss AG) was used to capture and analyze the images.

Gene set enrichment analysis (GSEA) and Kyoto Encyclopedia of Genes and Genomes (KEGG). Datasets of 1,170 patients with breast cancer from The Cancer Genome Atlas (TCGA) (https://cancergenome.nih.gov/) were analyzed using GSEA (4.0.3; gsea-msigdb.org/gsea/index.jsp). According to the median expression of EPN3 in TCGA, the patients were divided into two groups: EPN3 high expression group $(n=535)$ and low expression group $(n=535)$. The 'c2. cp.reactome.v7.2.symbols.gmt' gene set was downloaded from the Molecular Signature Database (http://software. broadinstitute.org/gsea/msigdb/index.jsp) and used for enrichment analysis. The analysis of each gene set was performed 1,000 times to obtain the normalized enrichment score. False discovery rate $<0.25$ and normal $\mathrm{P}<0.05$ were considered to indicate significant enrichment. The 'clusterProfiler' package (version 3.16.0), 'org.Hs.eg.db' package (version 3.11.4), 'enrichplot' package (version 1.8.1) and 'ggplot2' package (version 3.3.1) of $\mathrm{R}$ language (version 3.6.3; cran.r-project. org/bin/windows/base/old/3.6.3/) was used to perform KEGG pathway enrichment analysis.

Xenografts. Estradiol cypionate (drug soluble in corn oil, $2 \mathrm{mg} / \mathrm{kg}$ ) was administered subcutaneously every week from 1 week before administering the MCF7 cell line. Twelve female nude mice, aged 4-6 weeks, weighted 15-17 g, were selected for the experiment. Housing conditions were as follows: Temperature, $26-28^{\circ} \mathrm{C}$; relative humidity, $40-60 \%$; 10/14-h light/dark and access to food and water. In total, $\sim 3 \times 10^{6}$ cells in control group or shEPN3\#1 group were resuspended in $100 \mu \mathrm{l}$ PBS and injected into left breast fat pad of nude mice ( $n=6$ in each group). After the tumor was palpable, the length (L) and width (W) of the tumor were measured every 4 days. The formula for calculating the volume of tumor was $\left(\mathrm{L} \mathrm{x} \mathrm{W} \mathrm{W}^{2}\right) / 2$ (14). The duration of the experiment was 24 days. The investigators observed the mice daily to ensure animal welfare and to determine the humane endpoints, which 
A

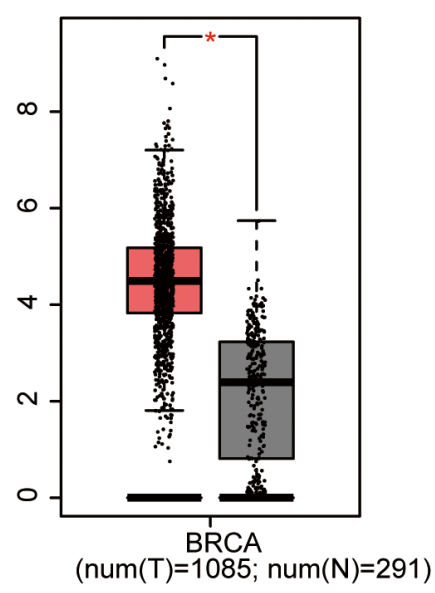

B

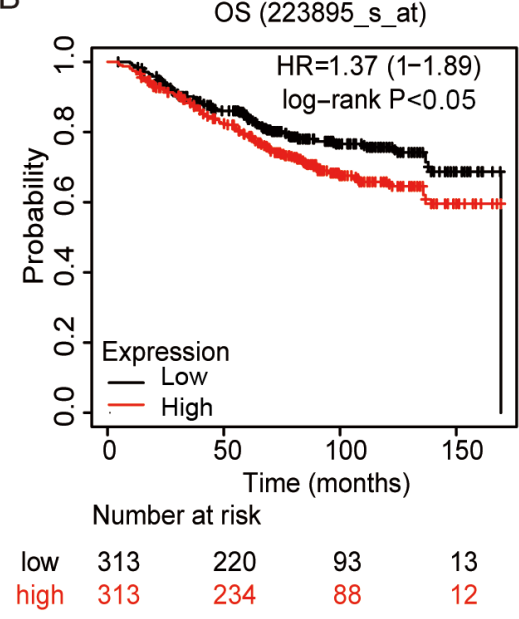

C

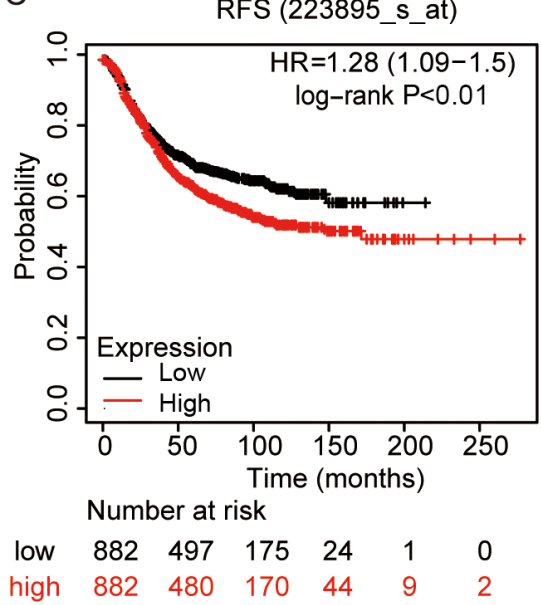

D

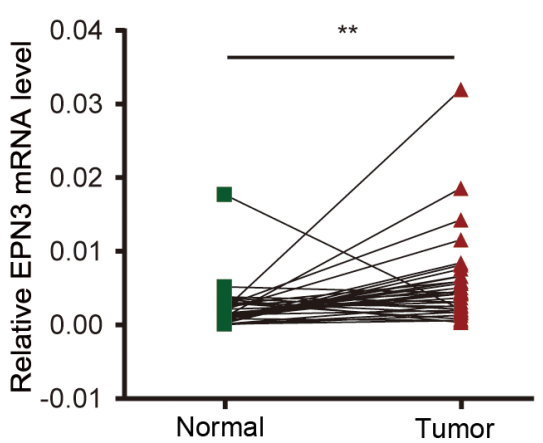

E

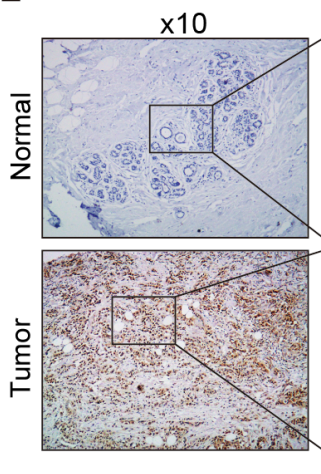

$\mathrm{F}$

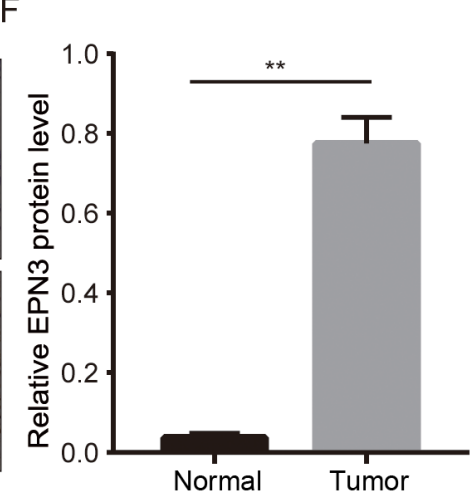

Figure 1. EPN3 is upregulated in breast cancer. (A) Analysis of EPN3 mRNA expression in normal breast tissues and breast cancer tissues in GEPIA. ${ }^{*} \mathrm{P}<0.05$. Kaplan-Meier analysis of (B) OS and (C) RFS in breast cancer cases with EPN3 low or high expression in Kaplan-Meier Plotter analysis. (D) Reverse transcription-quantitative PCR was used to assessed EPN3 mRNA expression in in breast cancer tissues and paired normal adjacent tissues. (E and F) Immunohistochemistry was used to assessed EPN3 protein expression in different tissues. Magnification, $\mathrm{x} 10$ and $\mathrm{x} 40$. $^{* *} \mathrm{P}<0.01$. OS, overall survival; RFS, release-free survival; HR, hazard ratio; EPN3, epsin 3.

included ruffled appearance, apathy, severe weight loss and tumor burden. The mice were sacrificed when they exhibited rapid weight loss $(>20 \%)$ and showed signs of deteriorating health due to the metastatic burden, such as hunching, dehydration and labored breathing. A total of 12 mice were used, with 6 mice in each group. All mice died of euthanasia. Mice were treated according to the Laboratory Animal Welfare (15). Mice were sacrifice via heart perfusion with $0.9 \%$ saline solution. The mice were anesthetized chloral hydrate $(400 \mathrm{mg} / \mathrm{kg}$; intraperitoneal), an upper abdominal incision was made and the heart was exposed. Then, $0.9 \%$ saline solution was injected into the apex of heart before cutting open the right auricle and pumped into the systemic circulation. No animals exhibited signs of peritonitis following the administration of $400 \mathrm{mg} / \mathrm{kg}$ chloral hydrate. When it was observed that the mice had stopped breathing, the mice were considered dead.

GEPIA and Kaplan-Meier Plotter analyses. The online GEPIA (version 1.0) database (http://gepia.cancer-pku.cn/) was used to analyze the expression level of EPN3 in breast cancer, and the online Kaplan-Meier Plotter (version 1.0) (https://kmplot. com/analysis/) was used to analyze the effect of EPN3 expression level on the overall survival (OS) time and recurrence-free survival (RFS) time of patients with breast cancer.
Statistical analysis. The results are presented as mean \pm SD. Paired or unpaired Student's t-test was used to conduct differential comparisons of two groups. One-way ANOVA was used to conduct differential comparisons of multiple groups, followed by Tukey's post hoc test. Statistical analyses were performed using GraphPad Prism 5.0 software (GraphPad Software, Inc.). All cell experiments are repeated three times independently. $\mathrm{P}<0.05$ was considered to indicate a statistically significant difference.

\section{Results}

EPN3 is upregulated in breast cancer. From the Online Kaplan-Meier Plotter database, it was found that the expression level of EPN3 in patients with breast cancer was higher compared with normal controls (Fig. 1A). Furthermore, through online Kaplan-Meier Plotter analysis, it was observed that the OS and RFS of patients with high EPN3 expression were significantly shorter compared with those with low EPN3 expression (Fig. 1B and C). These findings suggested that EPN3 was significantly associated with the survival and prognosis of patients with breast cancer. Furthermore, RT-qPCR and IHC were conducted to verify the mRNA and protein expression levels of EPN3 in breast cancer patient tissues. The 
A

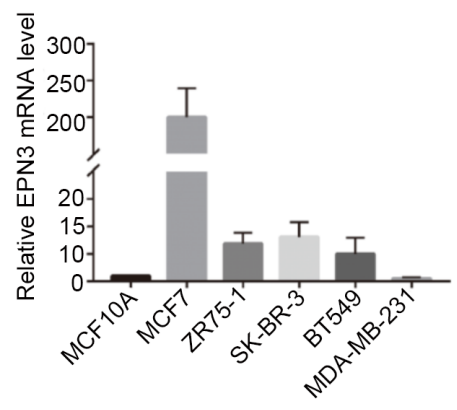

B

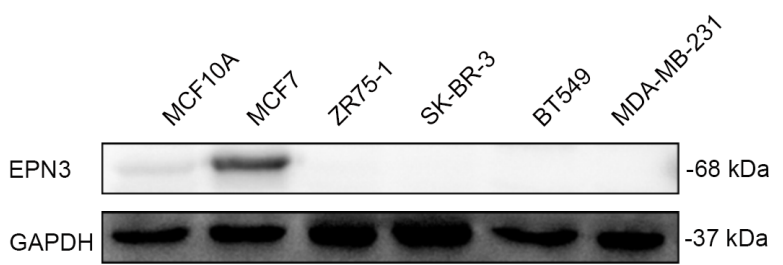

C

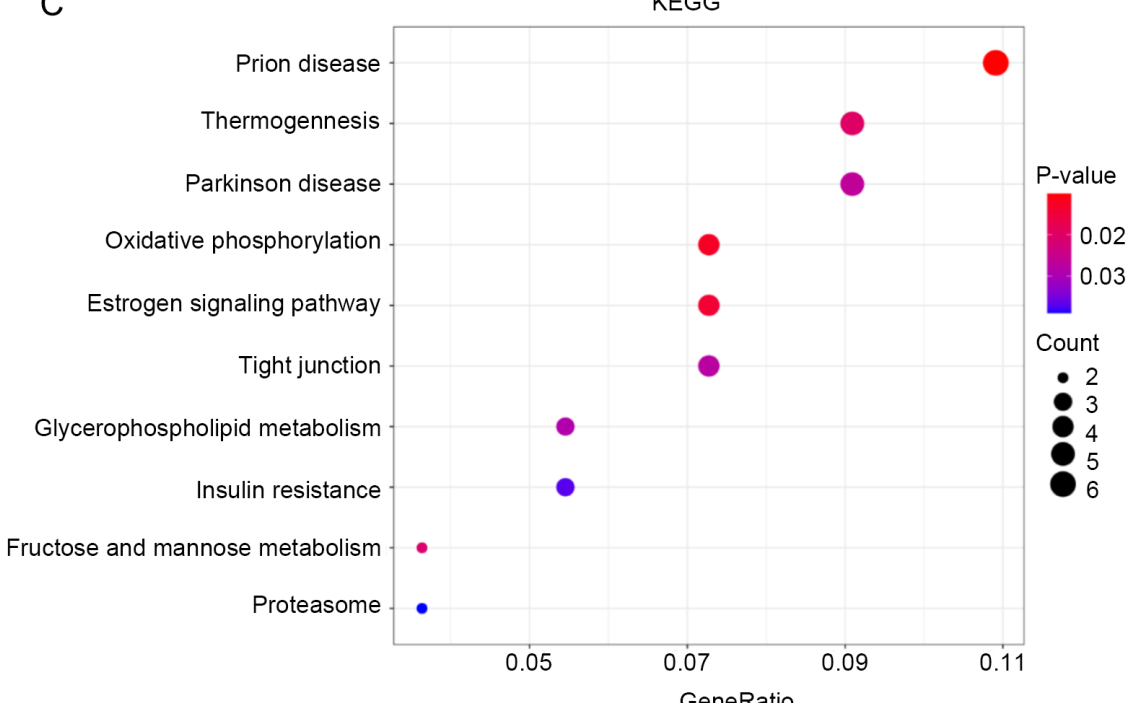

D

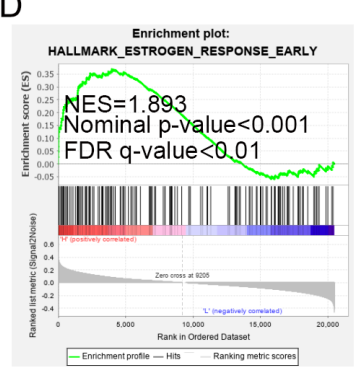

E

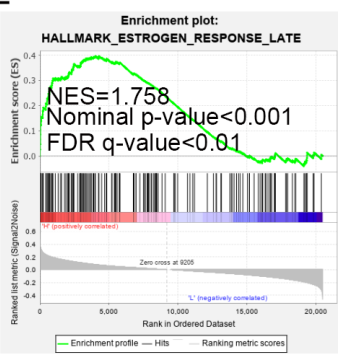

$\mathrm{F}$

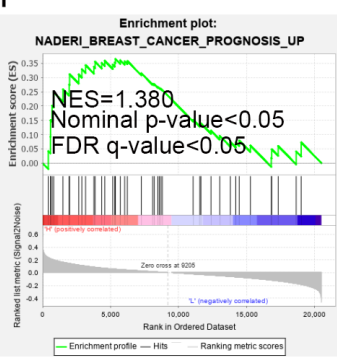

G

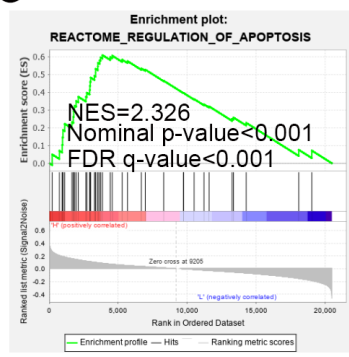

Figure 2. EPN3 is associated with estrogen receptor-positive breast cancer. (A) mRNA and (B) protein expression levels of EPN3 in breast cancer cell lines, as tested via reverse transcription-quantitative PCR and western blotting, respectively. (C) EPN3-related genes were enriched in pathways as determined via KEGG analysis. (D-G) Gene set enrichment analysis was used to analyze the signaling pathways enrichment in different groups. EPN3, epsin 3; FDR, false discover rate; NES, normalized enrichment score.

results demonstrated that the expression level of EPN3 in the tissues of patients with breast cancer was significantly higher compared with that in the adjacent breast tissues (Fig. 1D-F).

EPN3 may play an important role in ER-positive breast cancer. The expression level of EPN3 was also determined in different breast cancer cell lines, and it was found that EPN3 was significantly upregulated in the ER-positive breast cancer cell line MCF7, at both the mRNA and protein levels (Fig. 2A and B). The results also showed that EPN3 mRNA expression was high in T47D cells (data not shown). Moreover, KEGG analysis showed that EPN3-related genes were enriched in the 'estrogen signaling pathway' (Fig. 2C). The GSEA also indicated that EPN3 high expression was positively associated with the estrogen response (Fig. 2D and E). EPN3 was also associated with breast cancer prognosis and apoptosis (Fig. 2F and G). This suggests that EPN3 plays an important role in the occurrence and development of ER-positive breast cancer.

Effect of EPN3 knockdown on the proliferation and apoptosis of ER-positive breast cancer cells. To investigate the effect of EPN3 on the biological function of ER-positive breast cancer cells. A lentivirus infection system was used to infect MCF7 cells and knockdown EPN3 gene expression. Western blotting and RT-qPCR were used to detect the expression level of EPN3, and the results revealed that its protein and mRNA expression levels in the MCF7-shEPN3 group were significantly 

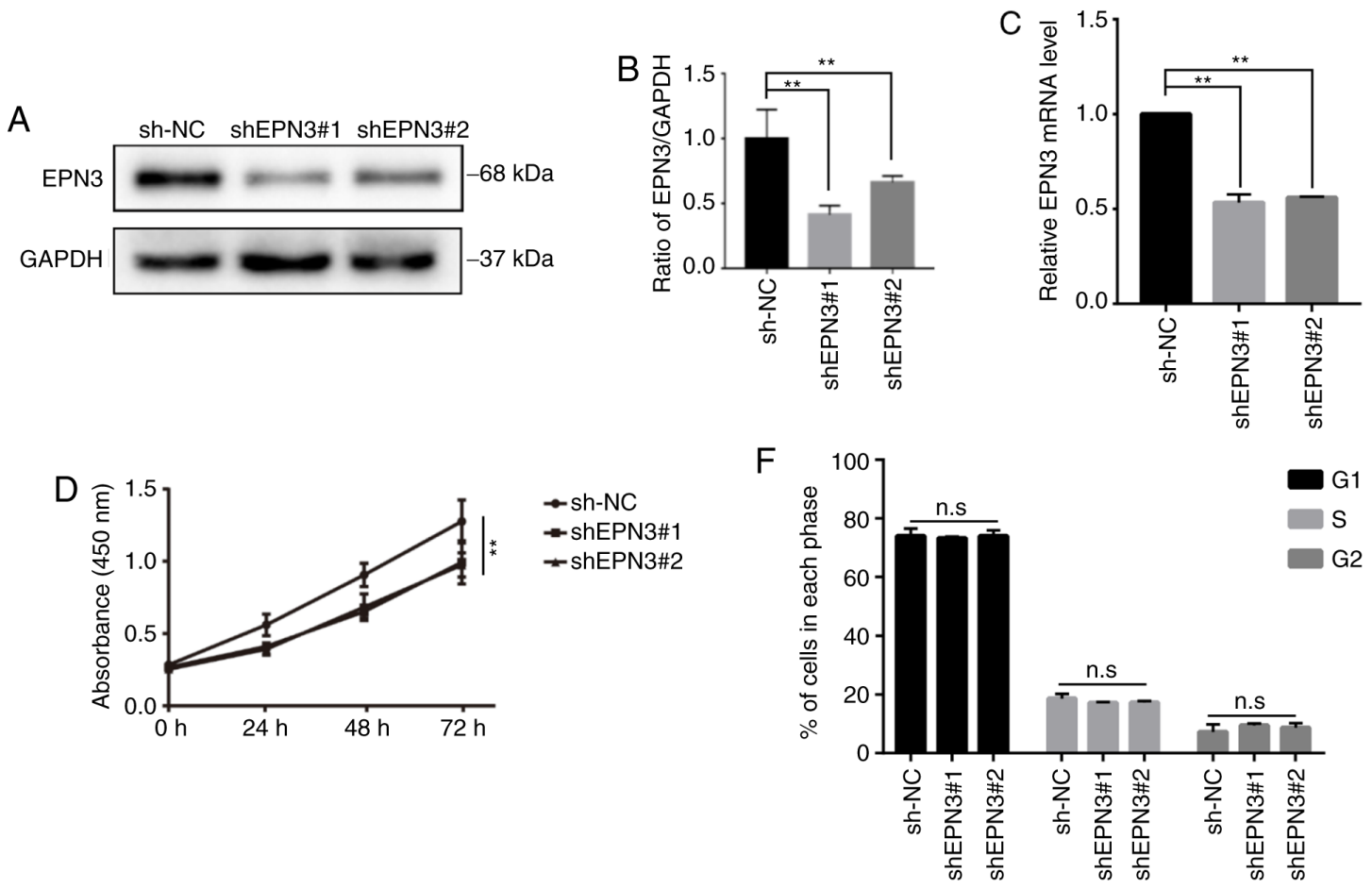
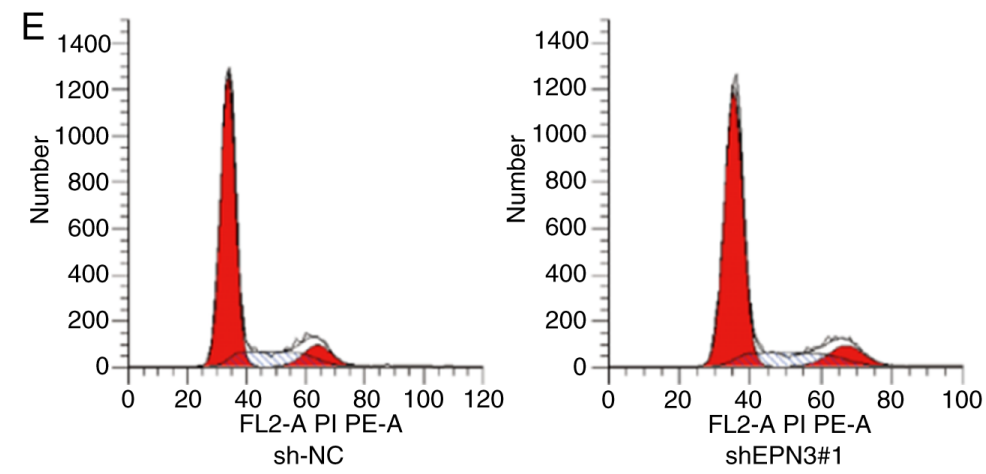

G
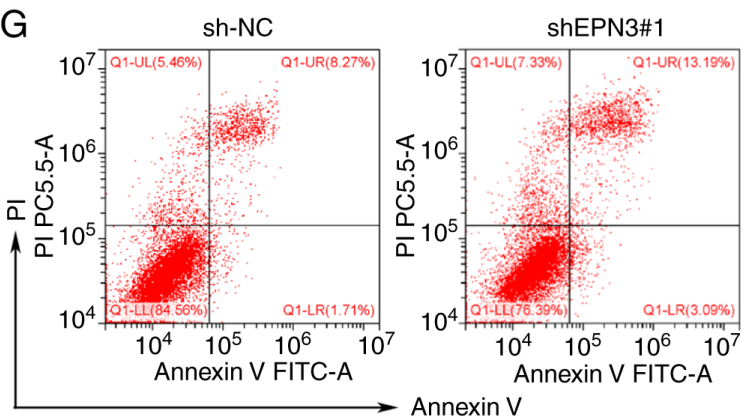

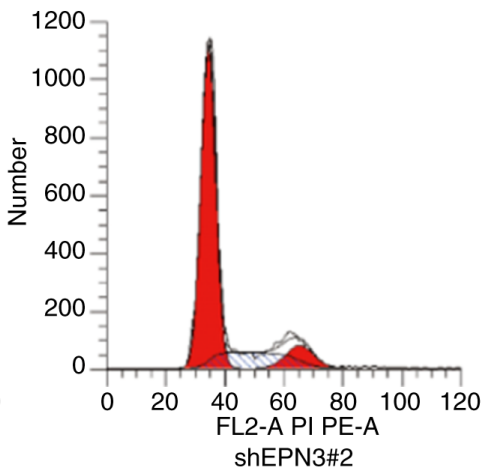

ShEPN3\#2

Figure 3. EPN3 promotes cell proliferation and inhibits apoptosis in breast cancer. (A and B) Western blotting and (C) reverse transcription-quantitative PCR analyses of EPN3 protein and mRNA expression in MCF7 cell transfected with shRNA1 and shRNA2 compared with NC. (D) Cell Counting Kit-8 assay was performed to examined cell proliferation. EPN3 knockdown suppressed the proliferation of MCF7 cells. (E and F) Flow cytometry was performed to assess the difference of cell cycle between shEPN3-MCF7 and NC-MCF7 cells. ( $\mathrm{G}$ and $\mathrm{H}$ ) Flow cytometry was performed to assess the difference in the apoptosis rate between shEPN3-MCF7 and NC-MCF7 cells. Data were represented as Mean $\pm \mathrm{SD} .{ }^{*} \mathrm{P}<0.05,{ }^{* *} \mathrm{P}<0.01$. n.s., not significant; NC, negative control; shRNA/sh, short hairpin RNA; EPN3, epsin 3.

lower compared with those in the sh-NC group (Fig. 3A-C), thereby indicating that the stable cell lines were successfully constructed.

CCK-8 and flow cytometry assays were used to detect the effect of EPN3 knockdown on the biological function of ER-positive breast cancer cells. The cell proliferation ability of the MCF7-shEPN3 group was significantly decreased compared with sh-NC group (Fig. 3D). Although there was no change in the cell cycle between the MCF7-shEPN3 group and sh-NC group (Fig. 3E and F), the apoptosis rate was significantly increased in MCF7-shEPN3 group (Fig. 3G and H). The effect of EPN3 knockdown on the production of downstream apoptotic proteins in breast cancer cell line, MCF7, was further examined. The results indicated that the protein expression 

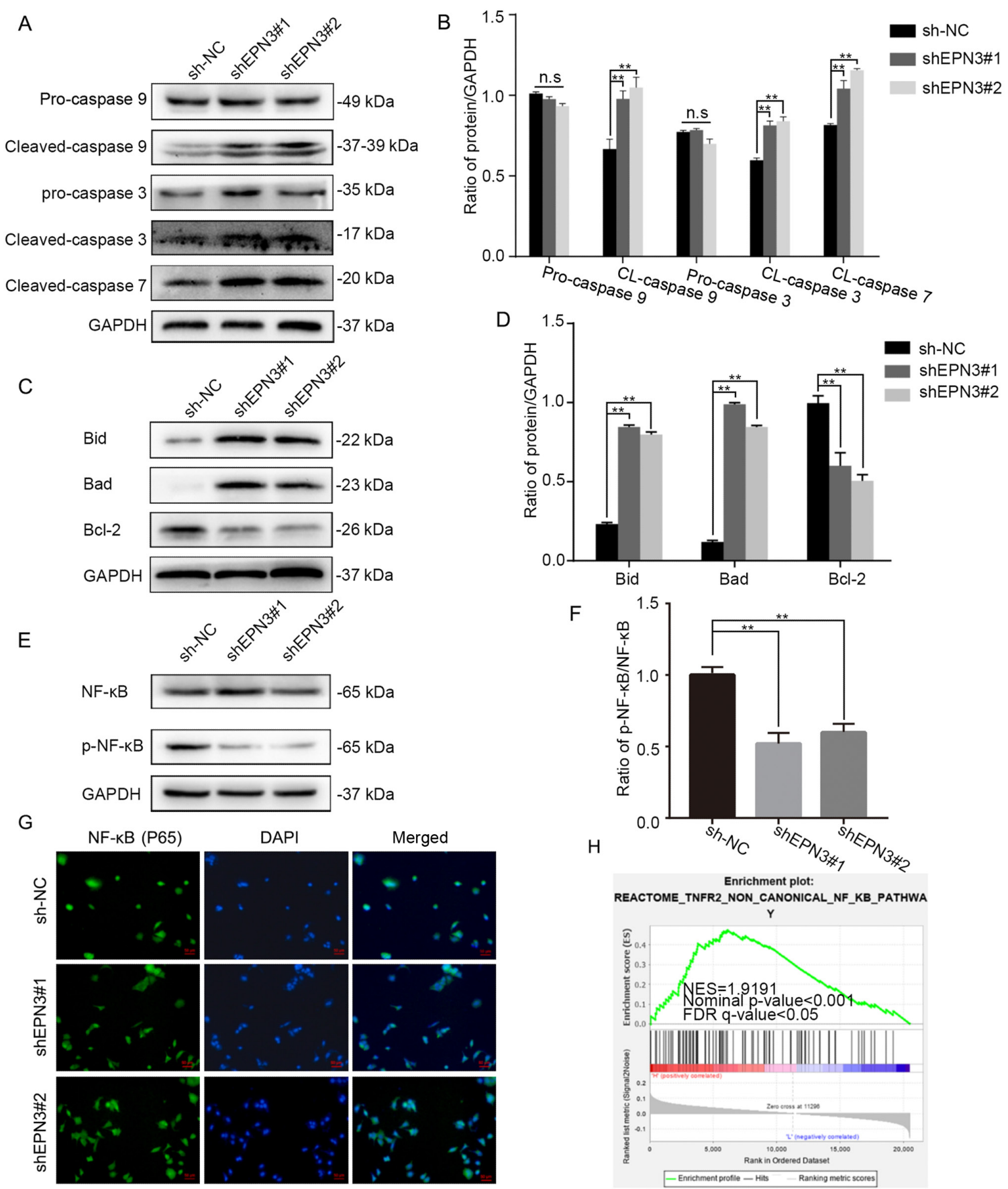

Figure 4. EPN3 facilitates NF-кB activation in breast cancer cells. Effects of EPN3 knockdown on the (A and B) caspase family and (C and D) Bcl-2 family were assessed via western blotting. (E and F) Effects of EPN3 knockdown on phosphorylation of NF- $\mathrm{B}$ (p65) were assessed via western blotting. (G) Immunofluorescent staining revealed that the nuclear translocation of NF-кB (p65) was decreased by EPN3 knockdown. Scale bar, $50 \mu \mathrm{m}$. (H) Gene set enrichment analysis was used to analyze the NF-kB pathway enrichment in high groups. ${ }^{* *} \mathrm{P}<0.01$. n.s., not significant; NC, negative control; shRNA/sh, short hairpin RNA; EPN3, epsin 3; p-, phosphorylated; FDR, false discover rate; NES, normalized enrichment score.

levels of cleaved caspase 9, cleaved caspase 3 and cleaved caspase 7 were significantly increased in the MCF7-shEPN3 group (Fig. 4A and B). With regards to the Bcl-2 family protein, compared with sh-NC group, Bid and Bad expression was significantly increased, while $\mathrm{Bcl}-2$ expression was significantly downregulated in the MCF7-shEPN3 group (Fig. 4C and D).

EPN3 may play a role in promoting cancer via the $N F-\kappa B$ pathway. To study the possible regulatory pathways EPN3 may participate in, this study used the 'c2.cp.reactome. v7.2.symbols.gmt' gene set to perform GSEA on 1,170 patients with breast cancer from TCGA database. These results suggested that EPN3 was positively correlated with the NF- $\kappa B$ pathway (Fig. 4H). The results of western blotting also indicated that the total NF- $\mathrm{KB}$ expression in breast cancer cells of the MCF7-shEPN3 group was not markedly altered, while the expression of $\mathrm{p}-\mathrm{NF}-\mathrm{\kappa B}$ protein was significantly downregulated (Fig. 4E and F). The effect of changes in EPN3 expression on NF- $\mathrm{KB}$ entering the nucleus was examined via 
A
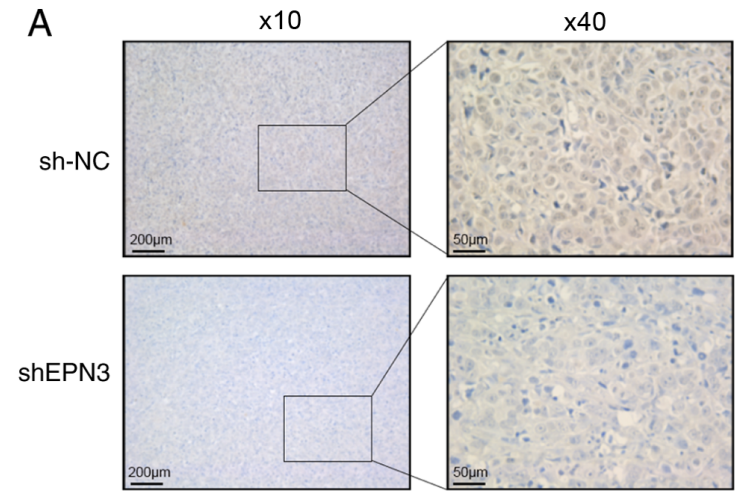

C

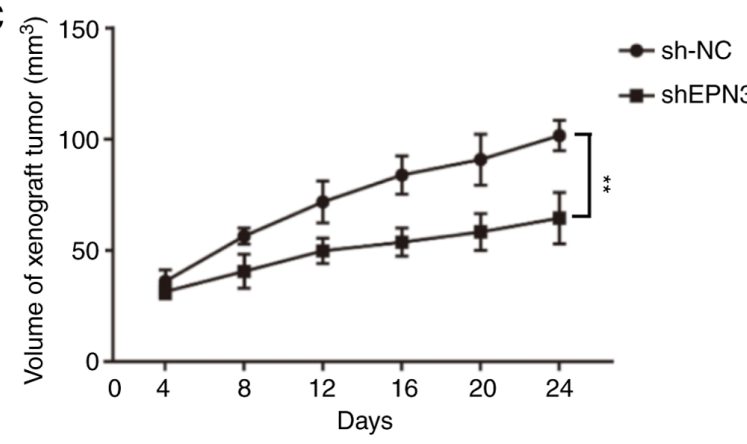

B
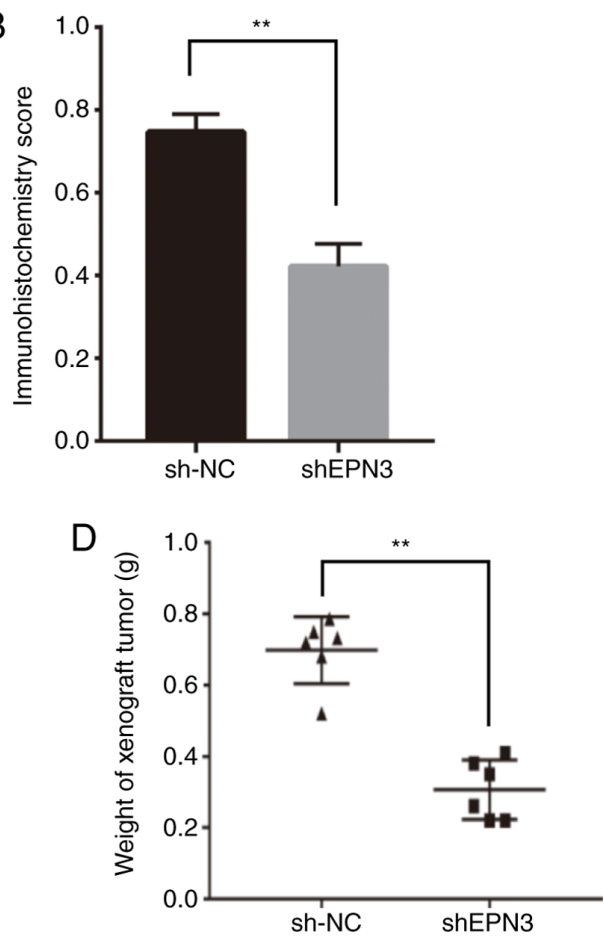

Figure 5. EPN3 knockdown inhibits tumor growth in vivo. (A) Immunohistochemistry was used to (B) assessed EPN3 protein expression between the two groups. Magnification, $\mathrm{x} 10$ and $\mathrm{x} 40$. (C) Tumor volume and (D) weight were compared between the two groups. ${ }^{* *} \mathrm{P}<0.01$. NC, negative control; shRNA/sh, short hairpin RNA; EPN3, epsin 3.

immunofluorescence. The results identified that the $\mathrm{NF}-\kappa \mathrm{B}$ fluorescence signal in the nucleus of the MCF7-shEPN3 group was weakened (Fig. 4G). Collectively, it was suggested that EPN3 may promote breast cancer cell proliferation and inhibit apoptosis by regulating NF- $\mathrm{B}$ nuclear translocation.

Xenograft tumor experiment. To further confirm that EPN3 promotes ER-positive breast cancer in vivo, the sh-NC MCF7 cells and MCF7-shEPN3\#1 cells were injected in situ into the breast fat pads of female nude mice. The size and weight of transplanted tumors in the MCF7-shEPN3\#1 group were significantly lower compared with those in the sh-NC group (Fig. 5C and D). The results of IHC revealed that the EPN3 protein expression in transplanted tumors in the MCF7-shEPN3\#1 group was significantly lower compared with that in the sh-NC group (Fig. 5A and B).

\section{Discussion}

ER-positive breast cancer accounts for $\sim 70 \%$ of breast cancer-related mortalities, and the recurrence rate remains high for several years after surgery (5). Although anti-estrogen therapy as a classic therapy has reduced the overall mortality of advanced breast cancer by $1 / 3$, the emergence of endocrine resistance affects the survival of patients with ER-positive breast cancer (16). The present results demonstrated that EPN3 expression was upregulated in breast cancer, and that the OS and RFS of patients with high EPN3 expression were significantly lower than those with low EPN3 expression, thereby confirming that EPN3 may be a risk marker for breast cancer. EPN3 has also been predicted to be associated with advanced metastasis of ER-positive breast cancer (17). Consistent with this previous study, the present results indicated that in the ER-positive breast cancer cell line MCF7, the mRNA and protein expression levels of EPN3 were significantly higher compared with those in other types of breast cancer cell lines. Pathway enrichment analysis also indicated that EPN3 was associated with estrogen. It is worth noting that ER-positive breast cancer cells have high expression of EPN3 in MCF7 cells, but not in ZR-75-1 cells. This contradictory result may be because the pathological classification of MCF7 cells is ER-positive and progesterone receptor $(\mathrm{PR})$ positive, while the pathological classification of ZR-75-1 cells is ER-positive and PR negative. Interestingly, the current study also detected the expression level of EPN3 in T47D cells with the same luminal A type, and the results showed that EPN3 mRNA expression was high in T47D cells (data not shown). Thus, it was suggested that EPN3 is highly expressed in luminal A breast cancer cells. These results indicated that EPN3 may specifically act on ER-positive breast cancer.

The EPN3 gene is located at 17q21.33. Although it is only $10.8 \mathrm{M}$ bp apart from the ERBB2 gene, the two appear to independently affect the tumor phenotype (11). EPN3 has also been identified as an independent risk marker for breast cancer (11). The earliest research on EPN3 reported that it was upregulated in the transitional keratinocytes of skin wounds (18). In mice, the deletion of the EPN3 gene did not lead to obvious pathological phenotypes in the systemic organs, which may be due to the overlapping functions of the other two epsin proteins (19). The role of EPN3 in tumor cells has been discovered, and it is involved in regulating tumor cell apoptosis and invasion, and is associated with the occurrence and development of tumors $(9,10,20-22)$. Previous studies also have reported 
that EPN3 regulates cell senescence and cell apoptosis $(10,21)$. Therefore, the current study infected MCF7 breast cancer cells with a lentiviral system to knockdown the expression of EPN3. The present results indicated that EPN3 knockdown inhibited the proliferation of MCF7 cells and induce their apoptosis. In addition, the effect of EPN3 knockdown on the downstream proteins of apoptosis was investigated, and it was found that pro-caspase 9 and pro-caspase 3 proteins in the caspase family were unchanged, while cleaved caspase 9, cleaved caspase 3 and cleaved caspase 7 were significantly upregulated. With regards to the Bcl-2 family, $\mathrm{Bcl}-2$ protein expression was significantly decreased, while Bid and Bad were increased. The changes in these proteins indicated the activation of the apoptosis cascade system (23). These results suggested that EPN3 promoted the proliferation of ER-positive breast cancer cells and inhibit apoptosis.

Next, GSEA was conducted to demonstrate that EPN3 was positively associated with the NF- $\kappa \mathrm{B}$ pathway. As previously reported, several factors such as p53, inhibitor of apoptosis protein (cytokine induced apoptosis inhibitor 1) and NF- $\kappa \mathrm{B}$ are involved in regulating the apoptosis pathway $(24,25)$. Song et al (26) observed that EPN1 and EPN2 can promote linear ubiquitination of NF- $\mathrm{B}$ essential modulators, leading to continuous $\mathrm{NF}-\mathrm{\kappa B}$ signaling and promoting the development of breast cancer. In addition, the activation of NF- $\kappa \mathrm{B}$ in ER-positive breast cancer is associated with endocrine and chemotherapy failure (27,28). Therefore, we hypothesized that EPN3's regulation of ER-positive breast cancer cell proliferation and apoptosis may be related to the activation of the $N F-\kappa B$ pathway. The present results indicated that knocking down the protein expression of EPN3 in MCF7 cells can downregulate the expression of $\mathrm{p}-\mathrm{NF}-\kappa \mathrm{B}$ (p-p65) protein, while the total protein of NF- $\kappa \mathrm{B}(\mathrm{p} 65)$ remained unchanged. At the same time, immunofluorescence also showed that the fluorescence signal of NF- $\kappa \mathrm{B}$ (p65) entering the nucleus was weakened. These results indicate that EPN3 can enhance the phosphorylation and activation of NF- $\kappa \mathrm{B}$ (p65), as well as increase its entry into the nucleus, activate the transcription of downstream target genes and promote the development of ER-positive breast cancer.

The limitation of the current study was that it did not analyze the interaction between EPN3 and ER. Since EPN3 belongs to the receptor family, it was speculated that EPN3 may regulate the endocytosis of ER- $\alpha$ in ER-positive breast cancer, or ER- $\alpha$ may in turn regulate the transcription and expression of EPN3.In future experiments, these issues will be discussed in detail.

In summary, the present study demonstrated that EPN3 was upregulated in ER-positive breast cancer, which can promote cell proliferation and inhibit cell apoptosis. The mechanism of action may be associated with the activation of the NF- $\mathrm{B}$ B signaling pathway. This study provides novel insights into the role of EPN3 in promoting the occurrence and development of breast cancer.

\section{Acknowledgements}

Not applicable.

\section{Funding}

This study was funded by the Natural Science Foundation of Chongqing (grant no. cstc2020jcyj-msxmX0600).

\section{Availability of data and materials}

The datasets analyzed during the current study are available in The Cancer Genome Atlas repository, https://cancergenome. nih.gov/.

\section{Authors' contributions}

QW designed and performed the experiments, analyzed the data and wrote the manuscript. QL and WZ analyzed the data and prepared figures. XZ and HL designed the experiments and supervised the study. XZ and HL confirm the authenticity of the raw data. All authors read and approved the final manuscript.

\section{Ethics approval and consent to participate}

The use of human specimen and animal experiment were approved by the Institutional Ethics Committees of The First Affiliated Hospital of Chongqing Medical University.

\section{Patient consent for publication}

Not applicable.

\section{Competing interests}

The authors declare that they have no competing interests.

\section{References}

1. Siegel RL, Miller KD and Jemal A: Cancer statistics, 2020. CA Cancer J Clin 70: 7-30, 2020.

2. Goldhirsch A, Wood WC, Coates AS, Gelber RD, Thürlimann B, Senn HJ and Members P: Strategies for subtypes-dealing with the diversity of breast cancer: Highlights of the St. Gallen international expert consensus on the primary therapy of early breast cancer 2011. Ann Oncol 22: 1736-1747, 2011.

3. Sotiriou C, Neo SY, McShane LM, Korn EL, Long PM, Jazaeri A, Martiat P, Fox SB, Harris AL and Liu ET: Breast cancer classification and prognosis based on gene expression profiles from a population-based study. Proc Natl Acad Sci USA 100: 10393-10398, 2003.

4. Liu B, Wang T, Wang H, Zhang L, Xu F, Fang R, Li L, Cai X, Wu Y, Zhang W and Ye L: Oncoprotein HBXIP enhances HOXB13 acetylation and co-activates HOXB13 to confer tamoxifen resistance in breast cancer. J Hematol Oncol 11: 26, 2018.

5. Early Breast Cancer Trialists' Collaborative Group (EBCTCG): Effects of chemotherapy and hormonal therapy for early breast cancer on recurrence and 15-year survival: An overview of the randomised trials. Lancet 365: 1687-1717, 2005.

6. Bykov VJN, Eriksson SE, Bianchi J and Wiman KG: Targeting mutant p53 for efficient cancer therapy. Nat Rev Cancer 18: 89-102, 2018.

7. De Camilli P, Chen H, Hyman J, Panepucci E, Bateman A and Brunger AT: The ENTH domain. FEBS Lett 513: 11-18, 2002.

8. Song K, Wu H, Rahman HN, Dong Y, Wen A, Brophy ML, Wong S, Kwak S, Bielenberg DR and Chen H: Endothelial epsins as regulators and potential therapeutic targets of tumor angiogenesis. Cell Mol Life Sci 74: 393-398, 2017.

9. Wang Y, Song W, Kan P, Huang C, Ma Z, Wu Q, Yao X and Zhang B: Overexpression of Epsin 3 enhances migration and invasion of glioma cells by inducing epithelial-mesenchymal transition. Oncol Rep 40: 3049-3059, 2018.

10. Mori J, Tanikawa C, Ohnishi N, Funauchi Y, Toyoshima O, Ueda K and Matsuda K: EPSIN 3, a novel p53 target, regulates the apoptotic pathway and gastric carcinogenesis. Neoplasia 19: 185-195, 2017. 
11. Schiano Lomoriello I, Giangreco G, Iavarone C, Tordonato C, Caldieri G, Serio G, Confalonieri S, Freddi S, Bianchi F, Pirroni S, et al: A self-sustaining endocytic-based loop promotes breast cancer plasticity leading to aggressiveness and pro-metastatic behavior. Nat Commun 11: 3020, 2020.

12. Subramaniyan B, Sridharan S, Howard CM, Tilley AMC, Basuroy T, de la Serna I, Butt E and Raman D: Role of the CXCR4-LASP1 axis in the stabilization of snail1 in triplenegative breast cancer. Cancers (Basel) 12: 2372, 2020.

13. Sun Q, Fan G, Zhuo Q, Dai W, Ye Z, Ji S, Xu W, Liu W, Hu Q, Zhang Z, et al: Pin1 promotes pancreatic cancer progression and metastasis by activation of NF- $\kappa$ B-IL-18 feedback loop. Cell Prolif 53: e12816, 2020

14. Feng X, Xu X, Xiao X, Zou K, Yu W, Wu J, Tang R, Gao Y, Hao J, Zhao X, et al: NMI inhibits cancer stem cell traits by downregulating hTERT in breast cancer. Cell Death Dis 8: e2783, 2017.

15. Jar AM: Animal welfare and the use of laboratory animals in scientific research. Rev Argent Microbiol 46: 77-79, 2014 (In Spanish).

16. Feng J, Wen T, Li Z, Feng L, Zhou L, Yang Z, Xu L, Shi S, Hou K, Shen J, et al: Cross-talk between the ER pathway and the lncRNA MAFG-AS1/miR-339-5p/CDK2 axis promotes progression of $\mathrm{ER}^{+}$breast cancer and confers tamoxifen resistance. Aging (Albany NY) 12: 20658-20683, 2020.

17. Hellwig B, Madjar K, Edlund K, Marchan R, Cadenas C, Heimes AS, Almstedt K, Lebrecht A, Sicking I, Battista MJ, et al Epsin family member 3 and ribosome-related genes are associated with late metastasis in estrogen receptor-positive breast cancer and long-term survival in non-small cell lung cancer using a genome-wide identification and validation strategy. PLoS One 11: e167585, 2016.

18. Spradling KD, McDaniel AE, Lohi J and Pilcher BK: Epsin 3 is a novel extracellular matrix-induced transcript specific to wounded epithelia. J Biol Chem 276: 29257-29267, 2001.

19. Ko G, Paradise S, Chen H, Graham M, Vecchi M, Bianchi F, Cremona O, Di Fiore PP and De Camilli P: Selective high-level expression of epsin 3 in gastric parietal cells, where it is localized at endocytic sites of apical canaliculi. Proc Natl Acad Sci USA 107: 21511-21516, 2010
20. Kohn KW, Zeeberg BM, Reinhold WC and Pommier Y: Gene expression correlations in human cancer cell lines define molecular interaction networks for epithelial phenotype. PLoS One 9: e99269, 2014.

21. Nagano T, Nakano M, Nakashima A, Onishi K, Yamao S, Enari M, Kikkawa U and Kamada S: Identification of cellular senescence-specific genes by comparative transcriptomics. Sci Rep 6: 31758, 2016.

22. Coon BG, Burgner J, Camonis JH and Aguilar RC: The epsin family of endocytic adaptors promotes fibrosarcoma migration and invasion. J Biol Chem 285: 33073-33081, 2010.

23. Arden $\mathrm{N}$ and Betenbaugh MJ: Life and death in mammalian cell culture: Strategies for apoptosis inhibition. Trends Biotechnol 22: 174-180, 2004.

24. Chang H, Li J, Qu K, Wan Y, Liu S, Zheng W, Zhang Z and Liu C: CRIF1 overexpression facilitates tumor growth and metastasis through inducing ROS/NFKB pathway in hepatocellular carcinoma. Cell Death Dis 11: 332, 2020.

25. Leo R, Therachiyil L, Siveen SK, Uddin S, Kulinski M, Buddenkotte J, Steinhoff $M$ and Krishnankutty AR: Protein expression profiling identifies key proteins and pathways involved in growth inhibitory effects exerted by guggulsterone in human colorectal cancer cells. Cancers (Basel) 11: 1478, 2019.

26. Song K, Cai X, Dong Y, Wu H, Wei Y, Shankavaram UT, Cui K, Lee $\mathrm{Y}$, Zhu B, Bhattacharjee $\mathrm{S}$, et al: Epsins 1 and 2 promote NEMO linear ubiquitination via LUBAC to drive breast cancer development. J Clin Invest 131: e129374, 2021.

27. Frasor J, El-Shennawy L, Stender JD and Kastrati I: NFкB affects estrogen receptor expression and activity in breast cancer through multiple mechanisms. Mol Cell Endocrinol 418 Pt 3: 235-239, 2015.

28. Smart E, Semina SE and Frasor J: Update on the role of $\mathrm{NF}_{\kappa} \mathrm{B}$ in promoting aggressive phenotypes of estrogen receptor-positive breast cancer. Endocrinology 161: bqaa152, 2020.

(i) (3) This work is licensed under a Creative Commons cc) Attribution-NonCommercial-NoDerivatives 4.0 International (CC BY-NC-ND 4.0) License. 